\title{
The non-receptor tyrosine kinase TNK2/ACK1 is a novel therapeutic target in triple negative breast cancer
}

\author{
Xinyan Wu ${ }^{1,2, *}$, Muhammad Saddiq Zahari ${ }^{1,2, *}$, Santosh Renuse ${ }^{1,2,5}$, Dhanashree \\ S. Kelkar ${ }^{1,2}$, Mustafa A. Barbhuiya ${ }^{2}$, Pamela L. Rojas ${ }^{1,2}$, Vered Stearns ${ }^{3}$, Edward \\ Gabrielson $^{3,4}$, Pavani Malla ${ }^{6}$, Saraswati Sukumar ${ }^{3}$, Nupam P. Mahajan $^{6,7}$, Akhilesh \\ Pandey ${ }^{1,2,3,4}$ \\ ${ }^{1}$ Department of Biological Chemistry, Johns Hopkins University School of Medicine Baltimore, MD 21205, U.S.A \\ ${ }^{2}$ McKusick-Nathans Institute of Genetic Medicine, Johns Hopkins University School of Medicine Baltimore, MD 21205, U.S.A \\ ${ }^{3}$ Department of Oncology, Johns Hopkins University School of Medicine Baltimore, MD 21205, U.S.A \\ ${ }^{4}$ Department of Pathology, Johns Hopkins University School of Medicine Baltimore, MD 21205, U.S.A \\ ${ }^{5}$ Institute of Bioinformatics, International Technology Park, Bangalore, 560066, India \\ ${ }^{6}$ Department of Drug Discovery, Moffitt Cancer Center, Tampa, FL 33612, U.S.A \\ ${ }^{7}$ Department of Oncologic Sciences, University of South Florida, Tampa, FL 33612, U.S.A \\ *These authors have contributed equally to this work \\ Correspondence to: Xinyan Wu, email: xinyan@jhmi.edu \\ Nupam P. Mahajan, email: Nupam.mahajan@moffitt.org \\ Akhilesh Pandey, email: pandey@jhmi.edu
}

Keywords: TNK2, triple negative breast cancer, tyrosine kinase, phosphorylation

Received: March 11,2016 Accepted: October 10,2016 Published: November 25, 2016

\section{ABSTRACT}

Breast cancer is the most prevalent cancer in women worldwide. About 15-20\% of all breast cancers do not express estrogen receptor, progesterone receptor or HER2 receptor and hence are collectively classified as triple negative breast cancer (TNBC). These tumors are often relatively aggressive when compared to other types of breast cancer, and this issue is compounded by the lack of effective targeted therapy. In our previous phosphoproteomic profiling effort, we identified the non-receptor tyrosine kinase TNK2 as activated in a majority of aggressive TNBC cell lines. In the current study, we show that high expression of TNK2 in breast cancer cell lines correlates with high proliferation, invasion and colony forming ability. We demonstrate that knockdown of TNK2 expression can substantially suppress the invasiveness and proliferation advantage of TNBC cells in vitro and tumor formation in xenograft mouse models. Moreover, inhibition of TNK2 with small molecule inhibitor $(R)-9 \mathrm{bMS}$ significantly compromised TNBC proliferation. Finally, we find that high levels of TNK2 expression in high-grade basal-like breast cancers correlates significantly with poorer patient outcome. Taken together, our study suggests that TNK2 is a novel potential therapeutic target for the treatment of TNBC.

\section{INTRODUCTION}

Breast cancer is a heterogeneous disease, with major subtypes categorized by expression of estrogen receptor (ER), progesterone receptor (PR) and HER2 receptor. Triple negative breast cancer (TNBC) is a subgroup of breast cancer cases that lack the expression of all these three receptors. TNBC accounts for $\sim 15 \%$ of invasive breast cancers and these cancers are often highly proliferative, poorly differentiated and associated with poor prognosis [1-4]. Unlike ER positive luminal breast cancers or breast cancers with HER2 amplification that can be treated with endocrine therapy [5] or HER2-targeted therapy [6], no targeted therapy is currently available for patients with TNBCs. A recent study found that several receptor tyrosine kinases including EGFR, MET and c-Kit were transcriptionally upregulated in different subsets of TNBCs $[7,8]$, suggesting that these upregulated receptor 
tyrosine kinases in TNBCs might be potential therapeutic targets for the treatment of TNBCs. However, clinical trials of targeting receptor tyrosine kinases in TNBCs with specific kinase inhibitors have, to date, produced mainly discouraging results. For example, treatment with monoclonal anti-EGFR antibody cetuximab, alone or in combination with cytotoxic chemotherapies, showed minimal improvement in progression-free and overall survival of TNBC patients $[9,10]$. These data suggest that molecules other than receptor tyrosine kinases may be involved in driving TNBCs or that one needs to further stratify the patients based on expression of individual molecular markers.

In our previous efforts to uncover the role of tyrosine kinase signaling pathways that are etiologically activated in TNBCs, we used quantitative mass spectrometry-based phosphoproteomics to globally profile the phosphotyrosine proteomes of a panel of 26 TNBC cell lines, leading to identification and quantitation of over 2,500 phosphopeptides [11]. Through systematic phenotypic characterization using invasion and soft agar colony formation assays of each cell line, we found that these TNBC cell lines were highly heterogeneous in terms of their growth rates and invasiveness. By correlating our phosphoproteomic data with oncogenic phenotypes, we identified the non-receptor tyrosine kinase, TNK2, as a protein that was hyperphosphorylated in a majority of aggressive TNBC cell lines, suggesting that TNK2 is a regulator of TNBC growth and proliferation.

TNK2, also known as ACK1 (activated Cdc42associated kinase), is a non-receptor tyrosine kinase which has been shown to be frequently amplified or mutated in multiple human cancers including breast, esophageal, lung, ovarian, pancreatic and prostate cancer $[12,13]$. Overexpression of TNK2 in cancer cell lines can increase the invasive phenotype, both in vitro and in vivo, resulting in increased mortality of a mouse model of metastasis $[13,14]$. Clinically, it was observed that amplification of the $T N K 2$ gene in primary tumors correlates with poor prognosis [13]. Further, expression of activated TNK2 was positively correlated with the severity of disease progression, and inversely correlated with the survival of breast cancer patients [15]. Being a cytoplasmic kinase, TNK2 couples extracellular signals from multiple receptor tyrosine kinases including EGFR, HER2 and PDGFR to intracellular effectors [15-18].

It has been suggested previously that phosphorylation of TNK2 might correlate with breast cancer progression $[15,19]$; however, the functional significance of TNK2 expression and its role in breast cancer biology particularly in TNBCs - has not been well elucidated. Based on emerging importance of TNK2 in various malignancies, we undertook detailed characterization of this relatively understudied non-receptor tyrosine kinase. In the current study, we show that TNK2 is overexpressed in a majority of TNBC cell lines and TNK2 expression level is significantly associated with the aggressiveness of TNBCs. By performing genetic ablation studies, we have demonstrated that TNK2 expression not only drives TNBC proliferation but also confers invasiveness, which is reflected in tumor formation in xenograft mouse models. Further, analysis of breast cancer patient survival data revealed that high TNK2 expression is significantly correlated with worse outcome of high-grade TNBC patients. In addition, the importance of TNK2 signaling was also reflected in cell proliferation studies; treatment with TNK2-specific small molecule inhibitor, $(R)-9 \mathbf{b M S}$, significantly suppressed growth of multiple TNBC cell lines. Taken together, these data uncover dependence of TNBCs on TNK2 signaling for both proliferation and invasion capabilities, and offer the promise of TNK2 inhibition as a therapeutic strategy for a subset of aggressive TNBCs.

\section{RESULTS}

\section{Phenotype analyses correlate TNK2 levels with cellular aggressiveness}

In our previous study characterizing the activated tyrosine signaling pathways of 26 TNBC cell lines [11], we identified four phosphotyrosine sites of TNK2 (pY284, pY518, pY859 and pY860) in multiple TNBC cells lines (Figure 1A) and discovered that TNK2 pY518 is hyperphosphorylated in a majority of aggressive cell lines [11]. In order to examine the expression level of TNK2 in TNBC, we performed immunoblotting for TNK2 in our panel of 26 TNBC cell lines (Figure 1B). A significant fraction (15 out of 26) of TNBC derived cell lines exhibited medium to high levels of TNK2 expression. When we examined how TNK2 expression might be related to anchorageindependent growth and cellular invasion (as determined by colony formation and matrigel invasion assays, described in our previous study [11]), we observed that TNK2 expression levels were significantly correlated with anchorage-independent growth and cellular invasiveness, suggesting that TNK2 plays an important role in regulating oncogenicity of TNBC cells (Figure 1B). Interestingly, HBL100, one of the immortalized non-tumorigenic mammary epithelial cell line, shows a relative aggressive phenotype and high expression of TNK2 in our study. It has been shown that HBL100 cells express the oncogenic SV40 large T antigen [20]. Similar aggressive phenotype of HBL100 has also been reported in other studies [21,22].

To further test the role of TNK2 in regulating tumorigenicity, we performed siRNA knockdown of TNK2 in four TNBC cell lines with high expression levels of TNK2 (MDA-MB-435, HCC1806, HCC70 and $\mathrm{HCC} 1143)$ and three cell lines with low levels of TNK2 expression (BT549, HCC1937 and HCC1569). 
A

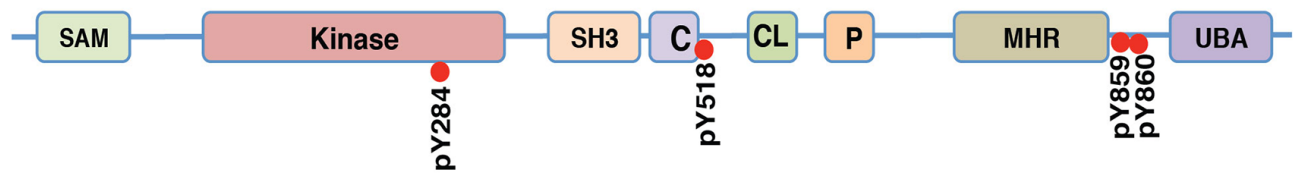

B
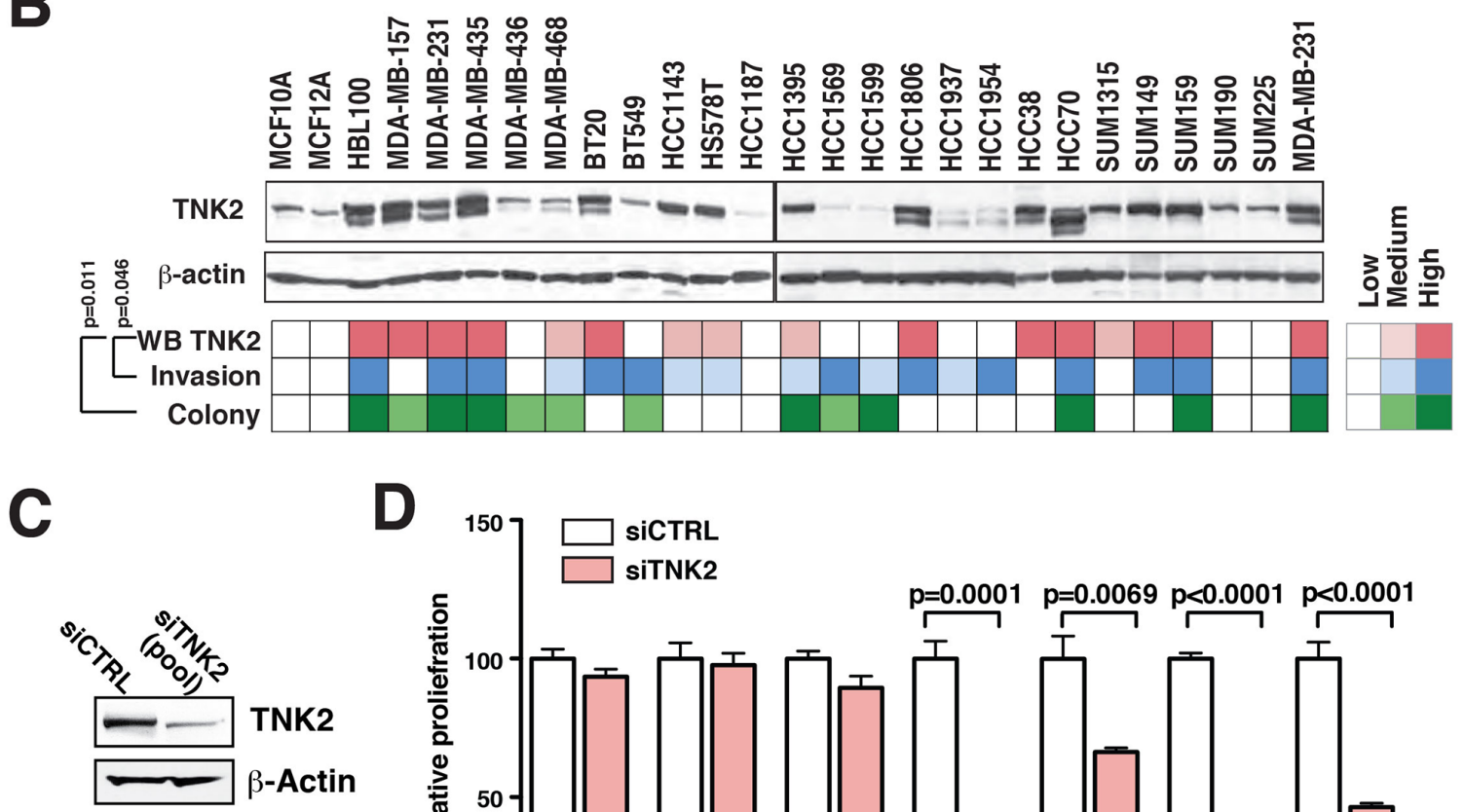

D

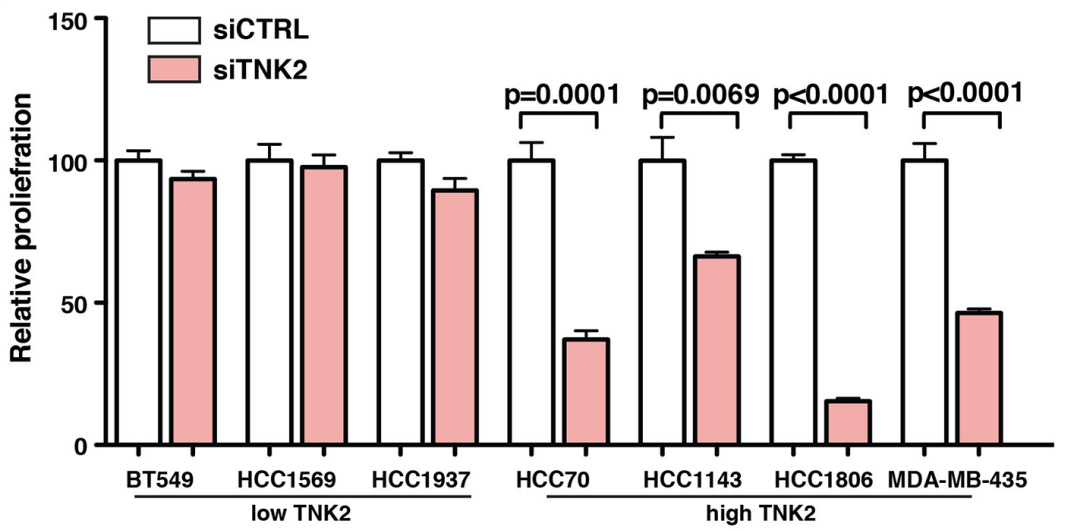

$\mathbf{E}$

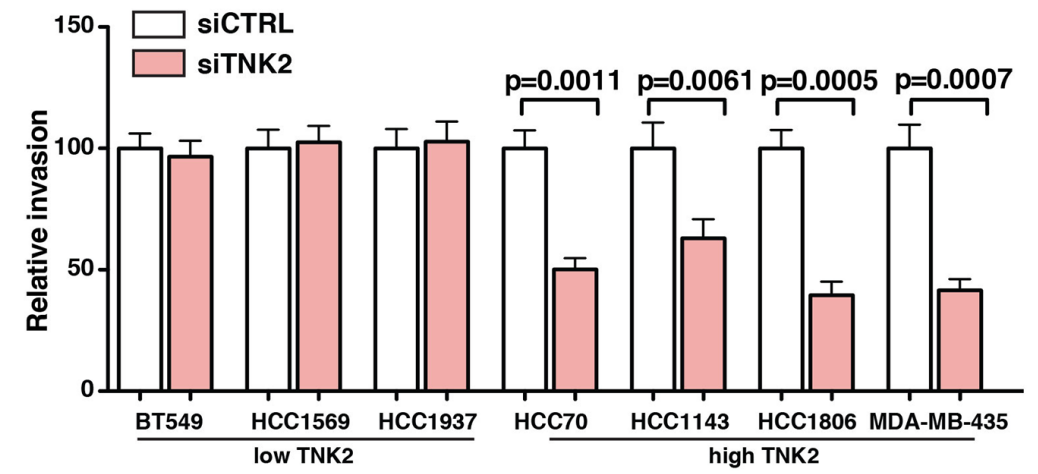

Figure 1: TNK2 is overexpressed in aggressive TNBC cell lines and required for oncogenic phenotype. A. A schematic representation of TNK2 domain architecture. Different structural domains present in TNK2 are shown. C, Cdc42-binding domain; CL, clathrin-interacting domain; kinase, tyrosine kinase domain; P, PPXY motif or WW domain-interacting region and SAM, sterile $\alpha$ motif. Tyrosine phosphorylation sites identified in our global phosphoproteomic study are specified. B. TNK2 expression level correlates with the aggressive phenotype of TNBC cells. Top panel: Western blot analysis to detect expression of TNK2 in the panel of TNBC cell lines. Color-coded plots showing the expression level of TNK2 (top row), invasiveness (middle row) and colony formation ability (bottom row) across the panel of TNBC cells. Spearman's rank correlation was performed for statistical analysis. C. TNK2 siRNA knockdown efficiency was examined in $\mathrm{HCC} 1395$ cells. $50 \mathrm{nM}$ siRNA were transfected in $\mathrm{HCC} 1395$ cells and an immunoblot was performed to evaluate the knockdown efficiency. $\beta$-Actin serves as the loading control. Proliferation D. and invasion assay $\mathbf{E}$. after knockdown using siRNA against TNK2 (siTNK2) or control siRNA (siCTRL) in TNBC cells that have low expression of TNK2 (BT549, HCC1569, HCC1937) or high expression of TNK2 (HCC70, HCC1143, HCC1806, MDA-MB-435). 
The SMARTPool siRNA containing four different siRNA targeting different TNK2 mRNA regions was able to efficiently knockdown TNK2 expression (Figure 1C). We then performed invasion and proliferation assays on these seven manipulated cell lines and found that, in cells expressing high TNK2 levels, proliferation and invasion of cells expressing high TNK2 levels were significantly reduced by knockdown of TNK2. However, the proliferation and invasiveness of the cell lines expressing low levels of TNK2 were not affected with TNK2 knockdown (Figure 1D, 1E and Supplementary Figure 1). These results suggest that high-expression/activation of TNK2 plays an important role in regulating aggressive phenotype of TNBC cells.

\section{TNK2 knockdown reduces aggressiveness of TNBC in vitro}

Dependence of TNBCs on TNK2 signaling opens up the possibility that genetic ablation of TNK2 in these cells could suppress their proliferation and invasion. To address the role of TNK 2 in aggressive breast cancers, we established a lentiviral-based Tet-ON inducible shRNA knockdown system to suppress TNK2 expression in a regulated fashion. The shTNK2 and scrambled control shRNA (shCTRL) expressing lentiviral particles were then used to infect HCC1395 and SUM159 cells - two highly aggressive TNBC cell lines with high basal expression of TNK2. Regulated induction of TNK2 knockdown with doxycycline was determined to be efficacious (Figure 2A, top panel). Upon induction by doxycycline, HCC1395shTNK2 and SUM159-shTNK2 cells showed significant reduction in colony formation in soft agar (Figure 2B). To calculate cell-doubling time for HCC1395 cells with or without induction of shTNK2, we performed quantitative cell proliferation assays. As shown in Figure 2C, almost no difference in cell proliferation was observed in HCC1395shCTRL cells regardless of doxycycline induction. However, doxycycline induction of HCC1395-shTNK2 cells substantially prolonged HCC1395 doubling time by $>2$-fold.

Subsequently, we studied the role of TNK2 on mammosphere formation, which is commonly used to indicate cellular stemness, an important tumorigenic hallmark in breast cancer. We found that upon induction of TNK2 shRNA, the cells completely lost their ability to form mammospheres (Figure 3A). This indicates that TNK2 expression is important for these cells to maintain their stem cell-like properties. To assess the potential effects of targeting TNK2 in vivo, we also performed 3-D Matrigel culture assays to better mimic in vivo physiological conditions. Similar to our mammosphere assay results, we observed that induction of TNK2 knockdown in HCC1395-shTNK2 cells completely eradicated colonies formed in the matrigel compared to HCC1395-shTNK2 with no induction and to HCC1395-
shCTRL cells (Figure 3B), supporting our hypothesis that TNK2 expression/activation is important for TNBC tumorigenesis in vivo.

We then tested whether knocking down TNK2 provided any synergistic activity with doxorubicin, a common chemotherapy agent. We observed that cells with TNK2 knock down by shRNA became rounder and started to die at a much lower dose of doxorubicin compared to cells that were treated with the same concentration of doxorubicin but were not induced to express TNK2 shRNA, which showed a more normal morphology (Figure $3 \mathrm{C})$. To determine if cells were undergoing apoptosis due to these treatments, we performed western blots to examine the apoptosis markers including the cleaved poly(ADP-ribose) polymerase 1 (PARP) and Caspase 8. As shown in Figure 3D, the levels of cleaved PARP and Caspase 8 were substantially increased in shTNK2 knockdown cells treated with doxorubicin compared to the cells without shTNK2 induction. Taken together, these results suggest a synergistic effect in reducing TNK2 expression with the treatment of chemotherapy agents such as doxorubicin.

\section{TNK2 knockdown reduces aggressiveness of TNBC in vivo}

To test the role of TNK2 in tumor growth in vivo, we established orthotopic xenograft using Tet-ON shTNK2 or shCTRL HCC1395 cells transplanted into mammary fat pads of NOD-SCID mice. shRNA (shTNK2 or shCTRL) expression was induced in vivo by doxycycline dissolved in drinking water. As shown in Figure 4A, tumor size in the doxycycline-induced shTNK2 group was significantly reduced as compared to the control group (Figure 4A, 4B). Western blot analysis for TNK2 protein expression confirmed the decrease in TNK2 expression in doxycycline-induced shTNK2 xenograft tumors (Figure 4C). Histopathological examination of the non-induced shTNK2 tumors demonstrated a lack of well-defined capsular margins, with cells pushing the borders for invasion compared to the shTNK2-induced tumors (Figure 4D). Immunohistochemical examination of tumor sections with Ki67, a marker of proliferation, and CD31, a marker of vascular endothelial cells, revealed that the shTNK2induced tumors indeed showed significantly lower extent of proliferation and vascularization (Figure 4D). Overall, our data shows that TNK2 plays important roles in conferring oncogenic phenotype in aggressive TNBCs in vivo.

\section{TNK2 levels correlate with poor outcome in patients}

To address the clinical relevance of TNK2 expression, we first examined the TNK2 expression levels in TCGA dataset using Oncomine concept analysis. As shown in Figure 5A, TNK2 mRNA expression level is 
A

HCC1395 ShCTRL ShTNK2

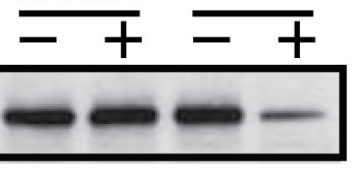

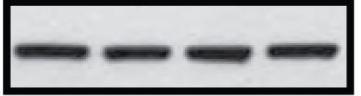

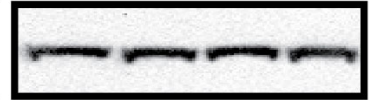

SUM159

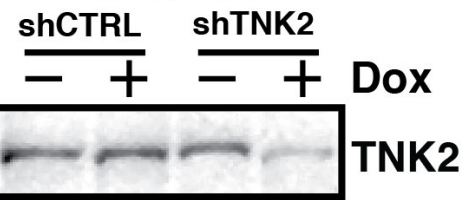

$\beta$-ACTIN

B
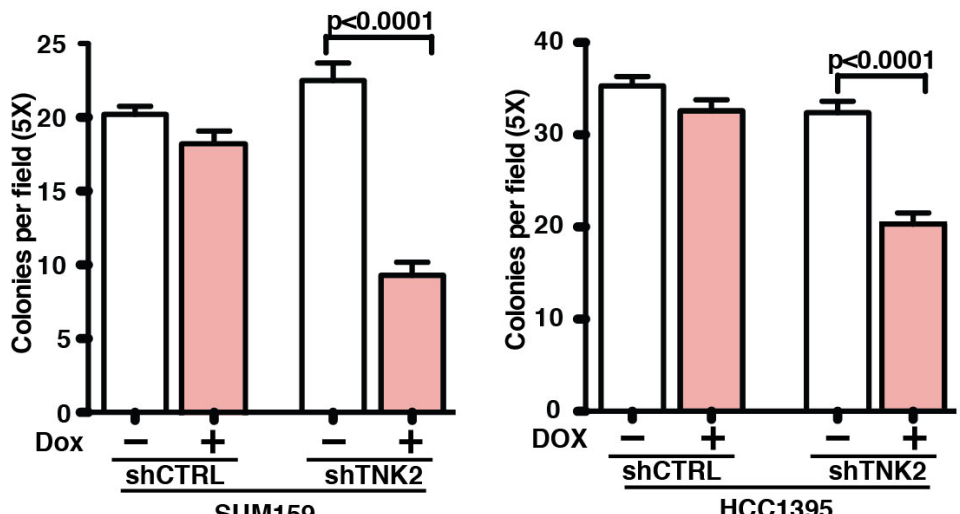

C
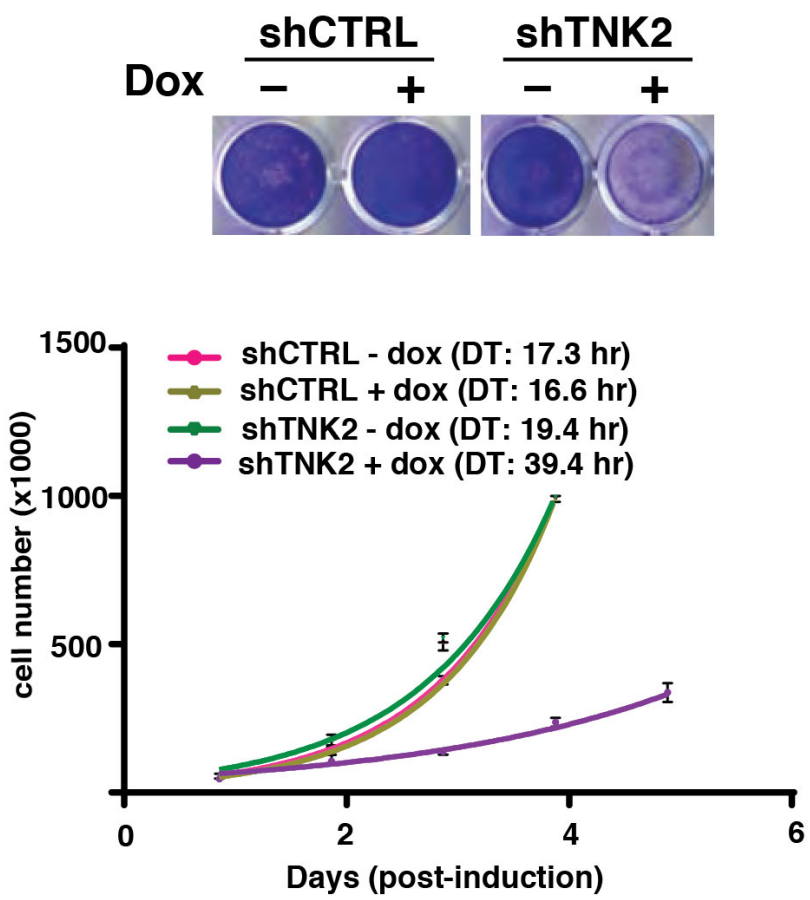

Figure 2: Inducible shRNA knockdown of TNK2 suppresses TNBC oncogenic phenotypes in vitro. A. Western blot analysis to examine TNK2 expression in HCC1395 and SUM159 cells with inducible TNK2 shRNA or scrambled control shRNA. $100 \mathrm{ng} / \mathrm{ml}$ doxycycline was used for induction. B. Colony formation assays with shTNK2/shCTRL transfected SUM159 and HCC1395 cells with or without doxycycline induction. Mann-Whitney tests were performed for statistical analyses. C. Cell proliferation assays with shTNK2 and shCTRL HCC1395 transfected cells with or without doxycycline induction. The cells were stained with crystal violet to visualize the difference in the number of cells (top) or the doubling time (DT) calculated for each condition (bottom). 

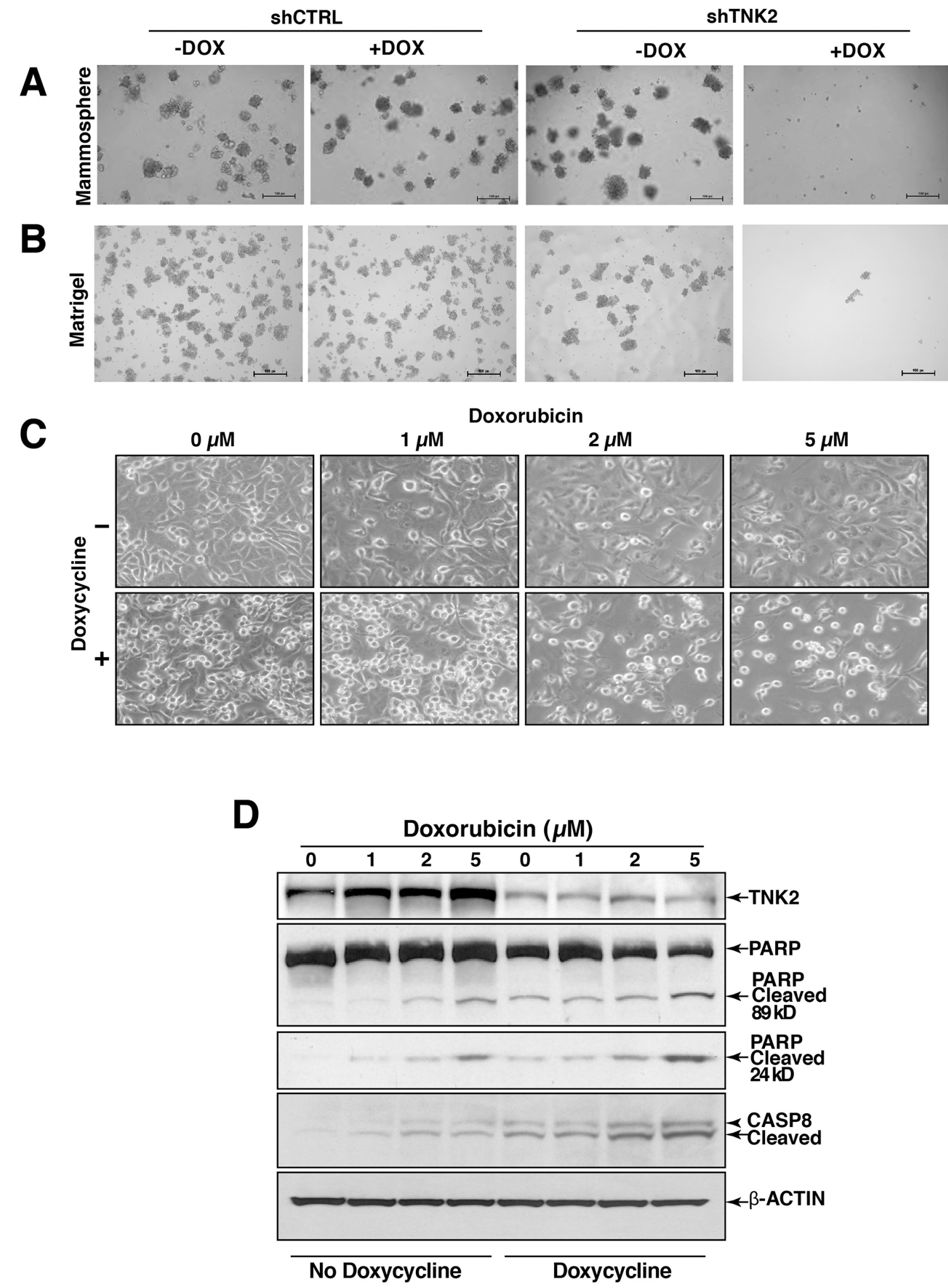

Figure 3: TNK2 is required for mammosphere formation. Mammosphere assays A. and 3D matrigel assays B. of shTNK2/ shCTRL HCC1395 cells with or without doxycycline induction. C. Synergistic effect of knocking down TNK2 with doxorubicin treatment. Doxycycline was used to induce shTNK2 expression. Induced or uninduced cells were treated with different doses of doxorubicin (as indicated). D. Western blot analysis to examine cleaved PARP and Caspase 8 in shCTRL and shTNK2 cells treated with doxorubicin and with or without doxycycline induction of shRNA. $\beta$-Actin serves as a loading control. 
lowest in normal breast and highest in TNBC comparing to other ER+ luminal and HER+ breast cancers. We also performed survival analysis using a publicly available gene expression database of breast tumors from 4,142 patients [23]. With our analysis focusing on the 669 tumors that were classified as basal-like breast cancers, we found no significant correlation between TNK2 expression and patient survival. However, when a subpopulation of 237 high grade basal-like breast cancer tumors were selected for the analysis, high TNK2 expression levels were significantly associated with poorer patient outcomes $(\mathrm{p}<0.01)$ (Figure 5B). It is also worth noting that TNK2 expression did not show a statistically significant correlation with clinical outcomes for patients with high grade ER-positive tumors (Figure 5C, 5D). We believe this clinical result when added to the results from our in vitro and in vivo studies provides strong evidence to support identifying TNK2 as a novel target for personalized therapy for patients with basallike/triple negative breast cancers that express high levels of this protein kinase.

\section{TNK2 kinase inhibition by small molecule inhibitor suppresses TNBC proliferation}

Ability of TNK2 to directly phosphorylate AKT at Tyr176 site leading to its activation [15] indicates that TNK2-AKT signalling nexus may be involved in driving
TNBC tumorigenicity. Recently, we have reported a new class of robust and soluble TNK2-specific small molecule inhibitor, $(R)-9 \mathbf{b M S}$ [24, 25]. To address the role of TNK2 in AKT Tyr176-phosphorylation in TNBCs, cells were treated with $(R)-9 \mathbf{b M S}$. A significant decrease in TNK2 activation, as seen by loss of TNK2 Tyr-phosphorylation is observed upon $(R)-9 b M S$ treatment (Figure 6A). Inhibition of TNK2 activation is also reflected in significant decrease in AKT Tyr176phosphorylation (Figure 6B). Further, several TNBC cell lines were found to be sensitive to the $(R)-9 \mathbf{b M S}$ treatment with $\mathrm{IC}_{50}$ of $0.45 \mu \mathrm{M}$ (MDA-MB-231), $0.48 \mu \mathrm{M}$ (MDA MB 157), $0.6 \mu \mathrm{M}$ (BT20), $0.67 \mu \mathrm{M}$ (SUM159), $0.75 \mu \mathrm{M}$ (HS578T) and $0.8 \mu \mathrm{M}$ (HCC38) and $1.75 \mu \mathrm{M}$ (HCC1395) (Figure 6C). Taken together, these data suggest that TNK2-AKT signaling may play an important role in TNBC growth and proliferation.

\section{DISCUSSION}

In our previous study, we investigated the intrinsic heterogeneous signaling networks present within the TNBC cell population [11]. In this study, as an extension to our previous work, we focused on a non-receptor tyrosine kinase, TNK2, which is an important signaling molecule involved in integrating signals from a number of different receptor tyrosine kinases [12]. Although TNK2 has also been reported to be amplified and mutated in

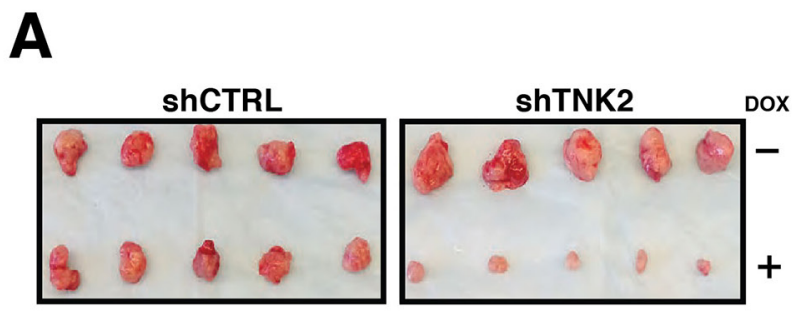

C

B
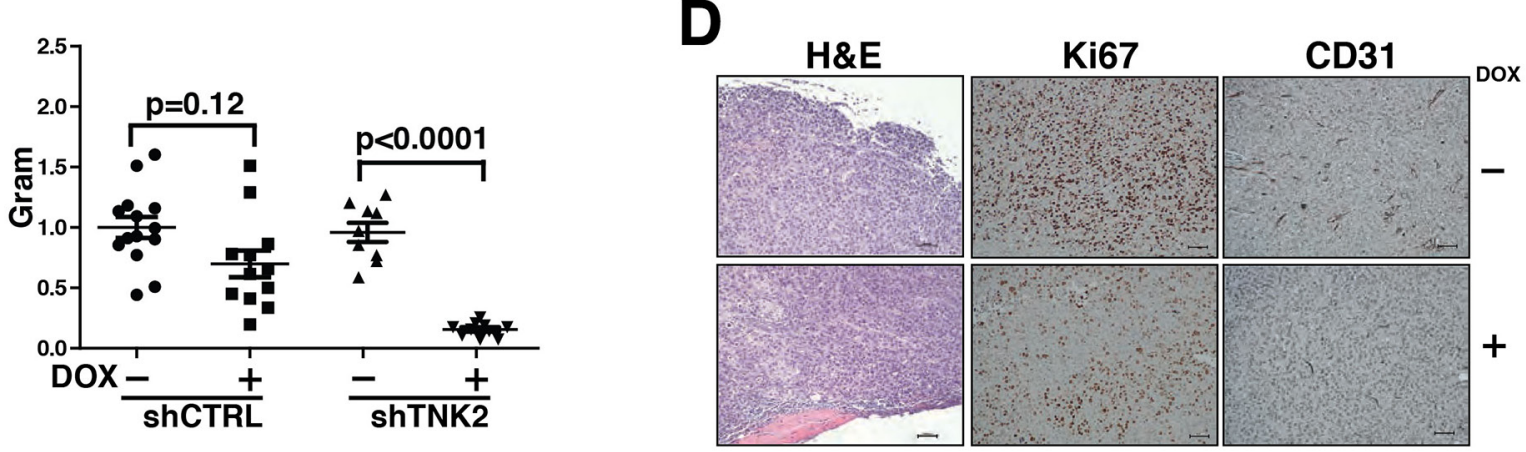

Figure 4: TNK2 knockdown suppresses tumor formation. A. Tumors harvested from mice injected with HCC1395-shTNK2 cells or HCC1395-shCTRL cells with or without doxycycline induction. B. Weight of tumors resected from mice xenografted with HCC1395-shCTRL or HCC1395-shTNK2 cells with or without doxycycline induction. P-values from Mann-Whitney tests to measure the statistical significance of size differences across the indicated groups are shown. C. Expression of TNK2 or beta actin detected by western blotting using specific antibodies in HCC1395 xenografted tumors is shown for the various tumors. D. Hematoxylin and eosin staining or immunohistochemical labeling for Ki67 and CD31 in HCC1395- shTNK2 xenografted tumors with (+) or without (-) induction of shTNK. 
multiple cancers [12], in breast cancer, its activation is likely to be facilitated by receptor tyrosine kinases that correlate with disease progression as well as survival.

Our global phosphotyrosine study identified several tyrosine phosphorylation sites in TNK2 (Figure $1 \mathrm{~A}$ ) in a number of TNBC cell lines and phosphorylation level of pY518 is elevated in TNBC cells with aggressive phenotypes [11]. The current study has revealed that TNK2 is not only hyperphosphorylated, but also overexpressed in many highly aggressive human TNBC tumors and also in TNBC cell lines, and that the expression of TNK2 correlates with aggressive phenotypes of TNBC cell lines. We also demonstrated that targeting TNK2 with specific siRNA/shRNA can substantially attenuate oncogenic phenotypes of TNBC cells in vitro and, more importantly, dramatically reduce tumor formation in preclinical xenograft mouse models, suggesting that these aggressive characteristics are driven by TNK2. Finally, our study shows promise for combining anti-TNK2 therapy with doxorubicin treatment for aggressive TNBCs, providing a pathway for future clinical applications of our findings.

Recent studies have shown that several ubiquitin ligases including NEDD4-1 [26], NEDD4-2 [27], SIAH1 and SIAH2 [28] can ubiquitinate TNK2 and induce TNK2 degradation. Of note, in ER positive breast cancer cells,

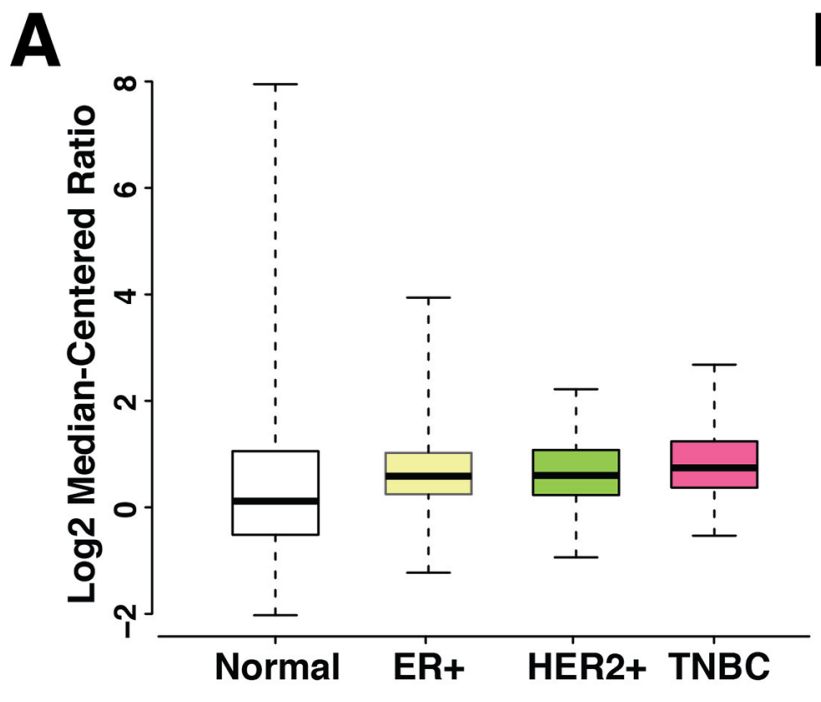

B

C

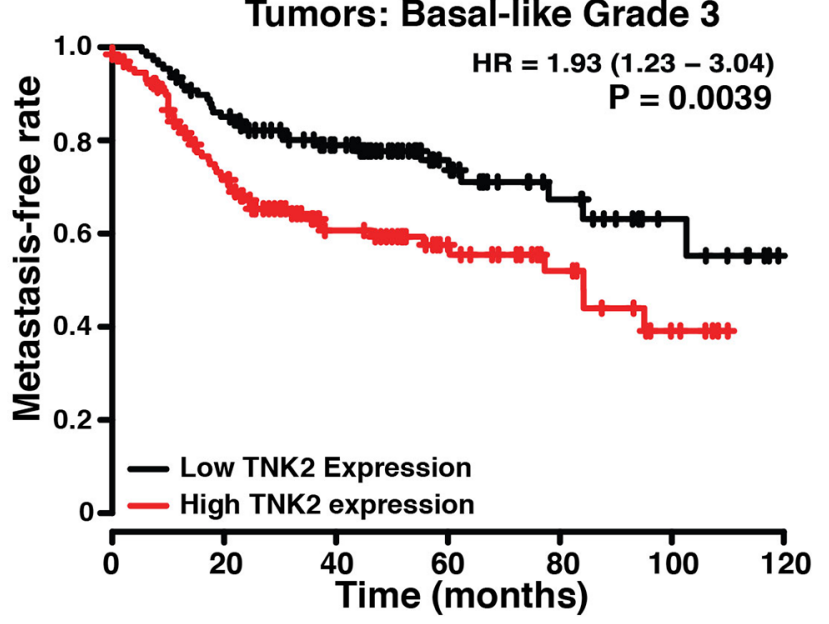

D
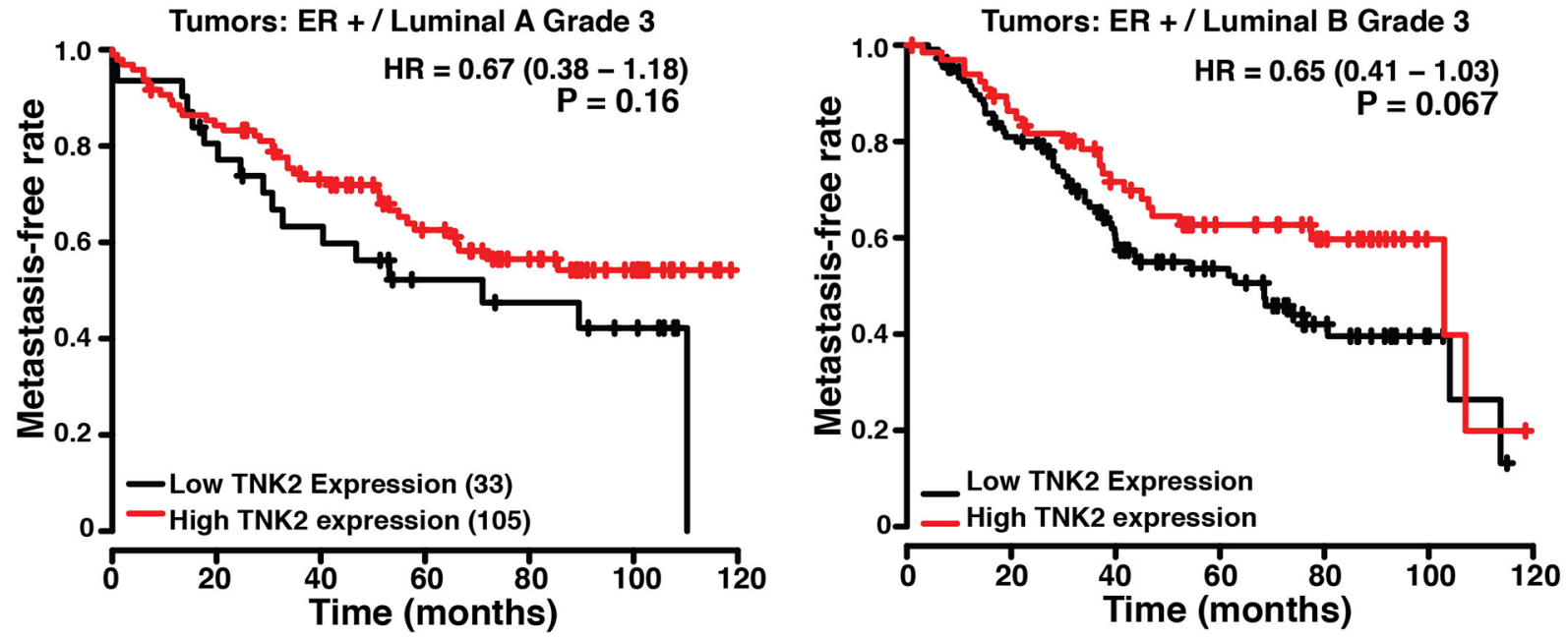

Figure 5: TNK2 levels correlate with poor outcome in patients. A. TNK2 expression level in normal breast and different subtypes of breast cancer. Expression data were retrieved from Oncomine using TCGA dataset and re-plotted with R package. B. Kaplan-Meier plot of 237 high-grade basal-like breast cancer patients stratified with high or low TNK2 gene expression. C-D. Kaplan-Meier plots of 138 highgrade luminal A (C) and 184 luminal B (D) breast cancer patients. The red line represents the survival curve of patients with high expression of TNK2 and the black line represents the surviving curve of patients with low expression of TNK2. 
A

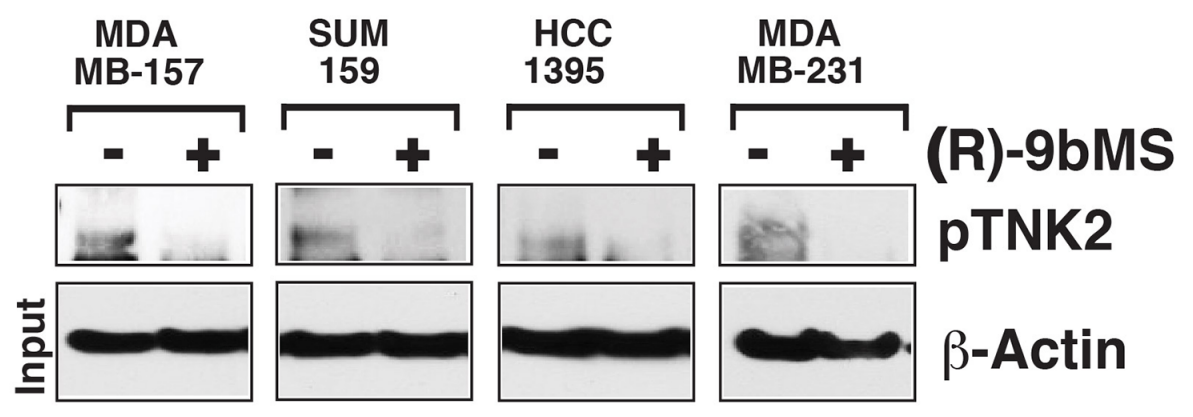

B
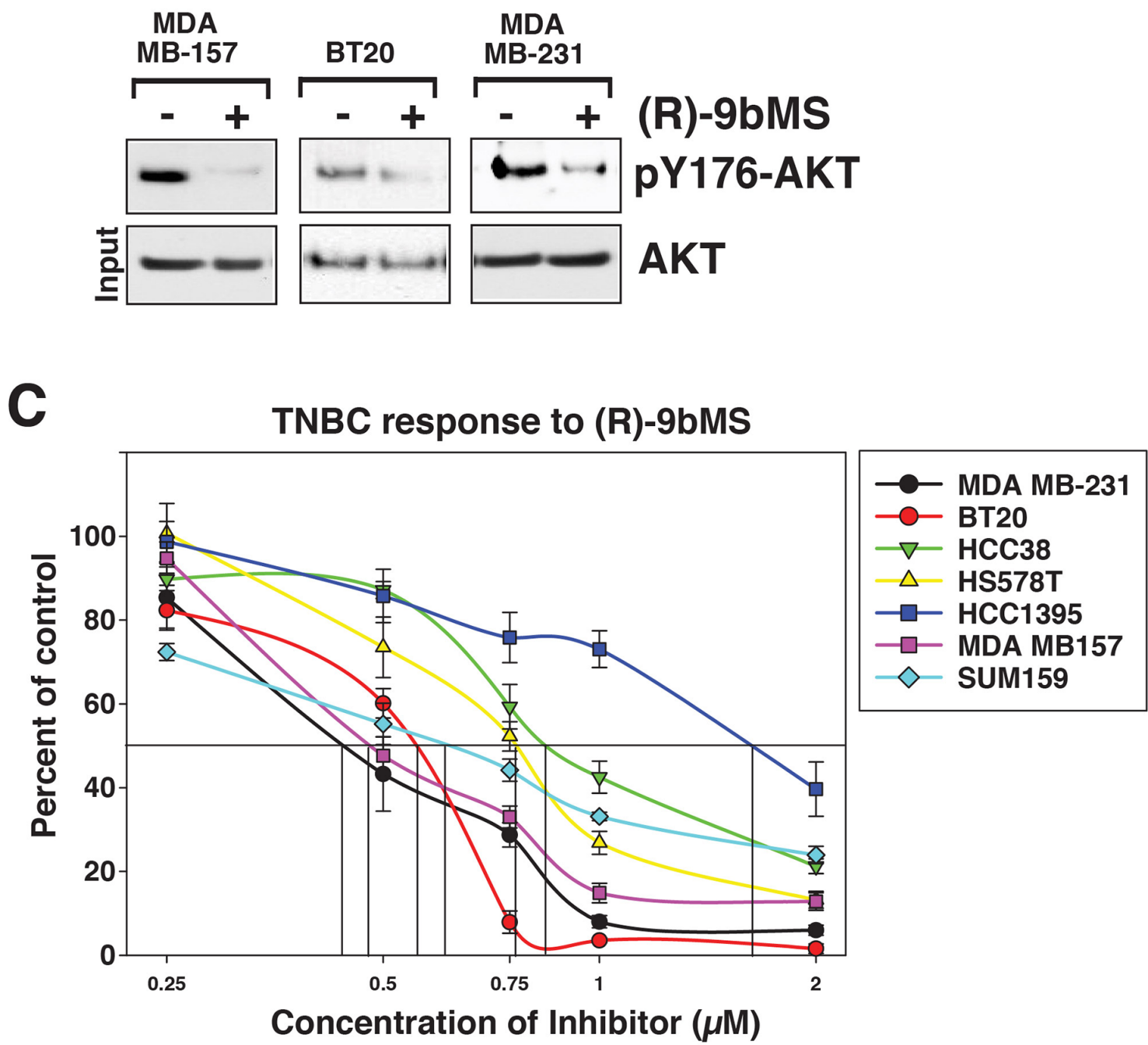

Figure 6: Small molecule inhibitor (R)-9bMS sensitizes TNK2 and its substrate AKT kinase phosphorylation compromising TNBC proliferation. A. TNBCs were treated with TNK2 inhibitor ( $5 \mu \mathrm{M}, 24$ hours); lysates were immunoprecipitated

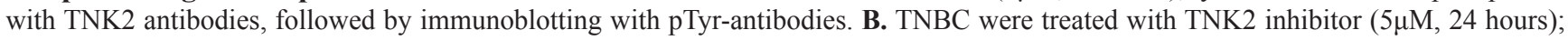
lysates were immunoprecipitated with pY176-AKT antibodies, followed by immunoblotting with pan AKT-antibodies C. TNK2 inhibitor $(R)-9 \mathrm{bMS}$ reduces TNBC growth. Cells were treated with $(R)-9 \mathrm{bMS}$ for $96 \mathrm{hr}$ and the number of viable cells were counted by trypan blue exclusion assay. Data represented as mean $\pm \operatorname{SEM}(n=2$, three replicates $)$. 
estrogen can activate ER and induce SIAH2 expression that subsequently ubiquitinates TNK2 and reduces TNK2 expression. Absence of ER in TNBC could be a potential reason why TNK2 is more expressed in TNBC (Figure 5A). A major downstream effector of TNK2 in hormonally regulated cancers has been AR [29], which also points to the potential of ER as another TNK2-interacting entity. However, TNBCs present a conundrum as although these cells lack expression of ER, they are exquisitely sensitive to loss of TNK2, suggesting that some other downstream effectors may be operational in TNBCs. TNK2 has been shown to interact with and directly phosphorylate AKT at an evolutionarily conserved tyrosine 176 residue, promoting AKT activation. Detection of AKT Tyr176phosphorylation in TNBCs (Figure 6A), its sensitivity to TNK2 inhibitor $(R)-9 \mathrm{bMS}$ which in turn compromising proliferation of TNBCs (Figure 6B) indicate that TNK2AKT signaling may drive TNBC tumor growth. Future studies may reveal whether sensitization of TNBCs by TNK2 inhibitor also involves other TNK2 effectors.

About $10-20 \%$ of breast cancers are found to be triple-negative. Due to limited treatment options that are currently available, there is intense interest in finding new therapies that can treat this subtype of breast cancer. This study not only provides evidence for 'addiction' of TNBCs to TNK2 signaling, but also provides a rationale for exploring TNK2-specific small molecule inhibitor $(R)$ $9 \mathrm{bMS}$ as a realistic treatment option for TNBC patients.

\section{MATERIALS AND METHODS}

\section{Cell culture}

Cell lines were obtained from ATCC and cultured in the appropriate media as previously described [11, 30, 31].

\section{Western blot and siRNA knockdown}

Each cell line was harvested and lysed in modified RIPA, and immunoblotting was performed as previously described [32]. The primary antibodies used in this study are anti-TNK2 (Santa Cruz Biotechnology), anti-PARP cleaved $89 \mathrm{Kd}$ protein (Cell Signaling), anti-PARP cleaved $24 \mathrm{kD}$ protein (Abcam), anti-cleaved Caspase 8 (Cell Signaling) anti-AKT (Cell Signaling), and anti-pY176-AKT antibody that was developed in our previous study [15]. $50 \mathrm{nM}$ siRNA targeting TNK2 (L-003102, SMARTpool, Dharmacon) was used for transfections with RNAiMax (Invitrogen). Cells were harvested 48 hours post-transfection for assessing knockdown efficiency or other follow-up experiments.

\section{Matrigel invasion assays}

Cells were washed once with PBS, detached using trypsin (Life Technologies) and $5 \times 10^{4}$ cells were seeded into Biocoat matrigel invasion chambers (BD Biosciences). Growth media supplemented with serum for each cell line was added in the lower wells as the chemoattractant. After 24 hours, the filter membranes were stained with DAPI (Invitrogen). The number of cells that penetrated through the matrigel and membrane was counted for ten randomly selected viewing fields at 20x magnification. ImageJ was used to count cell nuclei. Student's t-test was performed for statistical analysis.

\section{Soft agar colony formation assays}

Briefly, $0.5 \mathrm{ml}$ of $0.8 \%$ bottom layer agar was prepared in six-well plates. Cells with different treatments were separately trypsinized, centrifuged, resuspended in $0.4 \%$ agar medium (equal volumes of $0.8 \%$ agar and culture medium), and plated onto the top agar at 500 cells per well. The cells were grown for 14 days at $37^{\circ} \mathrm{C}$. Colonies were then stained with crystal violet and counted under the microscope. Mann-Whitney tests were performed to for statistical analyses.

\section{MTT cell proliferation assay}

MTT (3-(4, 5-dimethylthiazol-2-yl)-2, 5-diphenyltetrazolium bromide) assays were performed to measure cell proliferation. Briefly, cells that were transfected with different siRNAs in 96-well plate were left to grow for 5-7 days before the MTT assay. $1 \mathrm{mg} / \mathrm{ml}$ MTT in growth media was added into each well and the plate was incubated for two hours in $37^{\circ} \mathrm{C}$. Media was then removed and $100 \mu \mathrm{l}$ of DMSO and ethanol (1:1 by volume) was added into each well. The plate was then read for absorption at $530 \mathrm{~nm}$ on a microplate reader. Student's t-test was performed for statistical analysis.

To determine effect of TNK2 inhibitor, cells were treated with different concentration of $(R)-9 \mathbf{b M S}$ for 96 hours and live cells (determined by trypan blue exclusion) were counted.

\section{Generation of inducible shTNK2 cells}

A shRNA construct targeting a different TNK2 sequence (GGCAGUCAGAUCCUGCAUAAG) from that targeted by siRNA was designed and cloned into the pLKO-Tet-On-puro plasmid vector (Addgene) and the sequence was confirmed by Sanger sequencing. The plasmid was then packaged into viral particles by transfection into HEK293T cells along with packaging plasmids, psPAX2 and pMD2.G. After overnight transfection, the conditioned medium was collected and used to infect SUM159 and HCC1395 cells. Stably infected cells were selected by puromycin.

\section{In vivo tumor xenograft assays}

NOD-SCID mouse xenografts were performed as previously reported [33]. Briefly, 20 four to six week old female NOD-SCID mice were purchased from NCI. HCC1395 cells stably infected with Tet-ON inducible 
TNK2 shRNA or control shRNA were resuspended in matrigel:PBS (1:1 volume). $5 \times 10^{5}$ cells were injected onto the mammary fat pads of NOD-SCID mice at two sites per mouse. Mice were then either administered doxycycline in drinking water $(2 \mathrm{mg} / \mathrm{ml})$ to induce the expression of shRNA or normal water as control. Five mice were used for each treatment group. After four weeks, the mice were sacrificed and tumors were processed. Student's t test was performed for the statistical analysis to compare the tumor weight. All procedures were approved by Johns Hopkins University institutional Animal Care and Use Committee and were performed in accordance with the Animal Welfare Act regulations.

\section{Immunohistochemical labeling of xenograft tumors}

Immunohistochemical staining was performed on the formalin-fixed, paraffin-embedded xenograft tumors. Briefly, paraffin embedded slides were rehydrated and then treated with hydrogen peroxide to block endogenous peroxidase activity, and then washed with PBST buffer. Slides were then blocked with goat serum before diluted rabbit polyclonal antibodies to $\mathrm{Ki} 67$ and $\mathrm{CD} 31$ were added for protein binding at $4^{\circ} \mathrm{C}$ and incubated overnight. The slides were washed with PBST, incubated with biotinylated secondary antibody (Dako), and treated with DAB substrate kit (Dako) according to the manufacturer's instructions. The slides were then counter-stained using Mayer's Hematoxylin (Dako) before visualizing under the microscope.

\section{ACKNOWLEDGMENTS}

We thank the Department of Biotechnology of the Government of India for research support to the Institute of Bioinformatics, Bangalore, India. S. R. is a senior research fellow funded by University Grants Commission, Government of India and N.A.S is a senior research fellow funded by Council of Scientific and Industrial Research, Government of India. We thank the Majlis Amanah Rakyat (MARA) of Government of Malaysia for the research fellowship to M.S.Z. We thank the Mass Spectrometry and Proteomics Facility at Johns Hopkins University for their assistance.

\section{GRANT SUPPORT}

This study was supported by a NCI's Clinical Proteomic Tumor Analysis Consortium initiative (U24CA160036 to A.P.), a Career Catalyst Award from Susan G. Komen foundation to X.W, the Avon Foundation (S.S., and V.S.); the Safeway Breast Cancer Research Foundation (to X.W.), the National Institutes of Health grants (1R01CA135328 to N.P.M and 1R01CA184165 to A.P.), W81XWH-14-1-0002 and W81XWH-14-1-0003 awards from the Department of Defense (to N.P.M), W81XWH-15-1-0311 and W81XWH-15-1-0312 award from the Department of Defense (to N.P.M and A.P).

\section{CONFLICTS OF INTEREST}

N.P.M. is named as inventors on a patent application no. $60 / 862,763$ and 62/200,084 titled 'Inhibitors of ACK1/ TNK2 Tyrosine Kinase'.

\section{REFERENCES}

1. Bauer KR, Brown M, Cress RD, Parise CA and Caggiano V. Descriptive analysis of estrogen receptor (ER)-negative, progesterone receptor (PR)-negative, and HER2-negative invasive breast cancer, the so-called triple-negative phenotype: a population-based study from the California cancer Registry. Cancer. 2007; 109:1721-1728.

2. Brenton JD, Carey LA, Ahmed AA and Caldas C. Molecular classification and molecular forecasting of breast cancer: ready for clinical application? Journal of clinical oncology. 2005; 23:7350-7360.

3. Carey L, Winer E, Viale G, Cameron D and Gianni L. Triple-negative breast cancer: disease entity or title of convenience? Nature reviews Clinical oncology. 2010; 7:683-692.

4. Morris GJ, Naidu S, Topham AK, Guiles F, Xu Y, McCue P, Schwartz GF, Park PK, Rosenberg AL, Brill $\mathrm{K}$ and Mitchell EP. Differences in breast carcinoma characteristics in newly diagnosed African-American and Caucasian patients: a single-institution compilation compared with the National Cancer Institute's Surveillance, Epidemiology, and End Results database. Cancer. 2007; 110:876-884.

5. Cochrane DR, Cittelly DM, Howe EN, Spoelstra NS, McKinsey EL, LaPara K, Elias A, Yee D and Richer JK. MicroRNAs link estrogen receptor alpha status and Dicer levels in breast cancer. Hormones \& cancer. 2010; 1:306-319.

6. Tambasco M, Eliasziw M and Magliocco AM. Morphologic complexity of epithelial architecture for predicting invasive breast cancer survival. Journal of translational medicine. 2010; 8:140.

7. Gobardhan PD, Elias SG, Madsen EV, van Wely B, van den Wildenberg F, Theunissen EB, Ernst MF, Kokke MC, van der Pol C, Borel Rinkes IH, Wijsman JH, Bongers V, van Gorp J and van Dalen T. Prognostic value of lymph node micrometastases in breast cancer: a multicenter cohort study. Annals of surgical oncology. 2011; 18:1657-1664.

8. Lehmann BD, Bauer JA, Chen X, Sanders ME, Chakravarthy AB, Shyr Y and Pietenpol JA. Identification of human triple-negative breast cancer subtypes and preclinical models for selection of targeted therapies. The Journal of clinical investigation. 2011; 121:2750-2767. 
9. Baselga J, Gomez P, Greil R, Braga S, Climent MA, Wardley AM, Kaufman B, Stemmer SM, Pego A, Chan A, Goeminne JC, Graas MP, Kennedy MJ, Ciruelos Gil EM, Schneeweiss A, Zubel A, et al. Randomized phase II study of the anti-epidermal growth factor receptor monoclonal antibody cetuximab with cisplatin versus cisplatin alone in patients with metastatic triple-negative breast cancer. Journal of clinical oncology. 2013; 31:2586-2592.

10. Carey LA, Rugo HS, Marcom PK, Mayer EL, Esteva FJ, Ma CX, Liu MC, Storniolo AM, Rimawi MF, Forero-Torres A, Wolff AC, Hobday TJ, Ivanova A, Chiu WK, Ferraro M, Burrows E, et al. TBCRC 001: randomized phase II study of cetuximab in combination with carboplatin in stage IV triple-negative breast cancer. Journal of clinical oncology. 2012; 30:2615-2623.

11. Wu X, Zahari MS, Ma B, Liu R, Renuse S, Sahasrabuddhe NA, Chen L, Chaerkady R, Kim MS, Zhong J, Jelinek C, Barbhuiya MA, Leal-Rojas P, et al. Global phosphotyrosine survey in triple-negative breast cancer reveals activation of multiple tyrosine kinase signaling pathways. Oncotarget. 2015; 6:29143-29160. doi: 10.18632/oncotarget.5020.

12. Mahajan K and Mahajan NP. ACK1/TNK2 tyrosine kinase: molecular signaling and evolving role in cancers. Oncogene. 2015; 34:4162-4167.

13. van der Horst EH, Degenhardt YY, Strelow A, Slavin A, Chinn L, Orf J, Rong M, Li S, See LH, Nguyen KQ, Hoey $\mathrm{T}$, Wesche $\mathrm{H}$ and Powers S. Metastatic properties and genomic amplification of the tyrosine kinase gene ACK1. Proceedings of the National Academy of Sciences of the United States of America. 2005; 102:15901-15906.

14. Mahajan NP, Whang YE, Mohler JL and Earp HS. Activated tyrosine kinase Ack1 promotes prostate tumorigenesis: role of Ack1 in polyubiquitination of tumor suppressor Wwox. Cancer research. 2005; 65:10514-10523.

15. Mahajan K, Coppola D, Challa S, Fang B, Chen YA, Zhu W, Lopez AS, Koomen J, Engelman RW, Rivera C, MuraokaCook RS, Cheng JQ, Schonbrunn E, Sebti SM, Earp HS and Mahajan NP. Ack1 mediated AKT/PKB tyrosine 176 phosphorylation regulates its activation. PloS one. 2010; 5:e9646.

16. Sohn MH, Kang MJ, Matsuura H, Bhandari V, Chen NY, Lee CG and Elias JA. The chitinase-like proteins breast regression protein-39 and YKL-40 regulate hyperoxiainduced acute lung injury. American journal of respiratory and critical care medicine. 2010; 182:918-928.

17. Elias DL. Oncoplastic surgery articles. Plastic and reconstructive surgery. 2010; 125:1294; discussion 1294-1295.

18. Gates MA, Tworoger SS, Eliassen AH, Missmer SA and Hankinson SE. Analgesic use and sex steroid hormone concentrations in postmenopausal women. Cancer epidemiology, biomarkers \& prevention. 2010; 19:1033-1041.
19. Mahajan K and Mahajan NP. Shepherding AKT and androgen receptor by Ack1 tyrosine kinase. Journal of cellular physiology. 2010; 224:327-333.

20. Caron de Fromentel C, Nardeux PC, Soussi T, Lavialle C, Estrade S, Carloni G, Chandrasekaran K and Cassingena R. Epithelial HBL-100 cell line derived from milk of an apparently healthy woman harbours SV40 genetic information. Experimental cell research. 1985; 160:83-94.

21. Gordon LA, Mulligan KT, Maxwell-Jones H, Adams M, Walker RA and Jones JL. Breast cell invasive potential relates to the myoepithelial phenotype. International journal of cancer. 2003; 106:8-16.

22. Petersen OW, Ronnov-Jessen L, Howlett AR and Bissell MJ. Interaction with basement membrane serves to rapidly distinguish growth and differentiation pattern of normal and malignant human breast epithelial cells. Proceedings of the National Academy of Sciences of the United States of America. 1992; 89:9064-9068.

23. Gyorffy B, Benke Z, Lanczky A, Balazs B, Szallasi Z, Timar J and Schafer R. RecurrenceOnline: an online analysis tool to determine breast cancer recurrence and hormone receptor status using microarray data. Breast cancer research and treatment. 2012; 132:1025-1034.

24. Lawrence HR, Mahajan K, Luo Y, Zhang D, Tindall N, Huseyin M, Gevariya H, Kazi S, Ozcan S, Mahajan NP and Lawrence NJ. Development of novel ACK1/TNK2 inhibitors using a fragment-based approach. J Med Chem. 2015; 58:2746-2763.

25. Mahajan K, Malla P, Lawrence H, Chen Z, Sinha C-K, Malik R, Shukla S, Kim J, Coppola D, Lawrence NJ, Mahajan NP. ACK1 regulates hisone H4Tyr88 phosphorylation and $\mathrm{AR}$ gene expression in castration resistant prostate cancer. 2016; Under Revision.

26. Lin Q, Wang J, Childress C, Sudol M, Carey DJ and Yang W. HECT E3 ubiquitin ligase Nedd4-1 ubiquitinates ACK and regulates epidermal growth factor (EGF)-induced degradation of EGF receptor and ACK. Molecular and cellular biology. 2010; 30:1541-1554.

27. Chan W, Tian R, Lee YF, Sit ST, Lim L and Manser E. Down-regulation of active ACK1 is mediated by association with the E3 ubiquitin ligase Nedd4-2. The Journal of biological chemistry. 2009; 284:8185-8194.

28. Buchwald M, Pietschmann K, Brand P, Gunther A, Mahajan NP, Heinzel T and Kramer OH. SIAH ubiquitin ligases target the nonreceptor tyrosine kinase ACK1 for ubiquitinylation and proteasomal degradation. Oncogene. 2013; 32:4913-4920.

29. Mahajan NP, Liu Y, Majumder S, Warren MR, Parker CE, Mohler JL, Earp HS and Whang YE. Activated Cdc42-associated kinase Ack1 promotes prostate cancer progression via androgen receptor tyrosine phosphorylation. Proceedings of the National Academy of Sciences of the United States of America. 2007; 104:8438-8443. 
30. Neve RM, Chin K, Fridlyand J, Yeh J, Baehner FL, Fevr T, Clark L, Bayani N, Coppe JP, Tong F, Speed T, Spellman PT, DeVries S, Lapuk A, Wang NJ, Kuo WL, et al. A collection of breast cancer cell lines for the study of functionally distinct cancer subtypes. Cancer cell. 2006; 10:515-527.

31. Kao J, Salari K, Bocanegra M, Choi YL, Girard L, Gandhi J, Kwei KA, Hernandez-Boussard T, Wang P, Gazdar AF, Minna JD and Pollack JR. Molecular profiling of breast cancer cell lines defines relevant tumor models and provides a resource for cancer gene discovery. PloS one. 2009; 4:e6146.
32. Zhong J, Kim MS, Chaerkady R, Wu X, Huang TC, Getnet D, Mitchell CJ, Palapetta SM, Sharma J, O’Meally RN, Cole RN, Yoda A, Moritz A, Loriaux MM, Rush J, Weinstock DM, et al. TSLP signaling network revealed by SILAC-based phosphoproteomics. Molecular \& cellular proteomics. 2012; 11:M112 017764.

33. van de Ven S, Wiethoff A, Nielsen T, Brendel B, van der Voort M, Nachabe R, Van der Mark M, Van Beek M, Bakker L, Fels L, Elias S, Luijten P and Mali W. A novel fluorescent imaging agent for diffuse optical tomography of the breast: first clinical experience in patients. Molecular imaging and biology. 2010; 12:343-348. 